\title{
STABILITY OF A DELAY DIFFERENCE SYSTEM
}

\author{
MIKHAIL KIPNIS AND DARYA KOMISSAROVA
}

Received 28 January 2006; Revised 22 May 2006; Accepted 1 June 2006

We consider the stability problem for the difference system $x_{n}=A x_{n-1}+B x_{n-k}$, where $A$, $B$ are real matrixes and the delay $k$ is a positive integer. In the case $A=-I$, the equation is asymptotically stable if and only if all eigenvalues of the matrix $B$ lie inside a special stability oval in the complex plane. If $k$ is odd, then the oval is in the right half-plane, otherwise, in the left half-plane. If $\|A\|+\|B\|<1$, then the equation is asymptotically stable. We derive explicit sufficient stability conditions for $A \simeq I$ and $A \simeq-I$.

Copyright (c) 2006 M. Kipnis and D. Komissarova. This is an open access article distributed under the Creative Commons Attribution License, which permits unrestricted use, distribution, and reproduction in any medium, provided the original work is properly cited.

\section{Introduction}

Our purpose is to investigate the stability of the system

$$
x_{n}=A x_{n-1}+B x_{n-k}
$$

where $A, B$ are $(m \times m)$ real matrixes, $x_{n}: \mathbb{N} \rightarrow \mathbb{R}^{m}$, a positive integer $k$ is a delay. The structure of the solution of equation $x_{n}=A x_{n-1}+B x_{n-k}+f_{n}$ with commutative matrixes $A, B$ is considered in [6]. A similar scalar equation

$$
x_{n}=a x_{n-1}+b x_{n-k},
$$

where $a, b$ are real numbers, was studied by Kuruklis [8] and Papanicolaou [15]. The following assertion describes the boundaries of the stability domain for $(1.2)$ in the $(a, b)$ plane (see also Figure 1.1).

Theorem 1.1 (see [7, Theorem 2]). The zero solution of (1.2) with $k>1$ is asymptotically stable if and only if the pair $(a, b)$ is the internal point of the finite domain bounded by the 


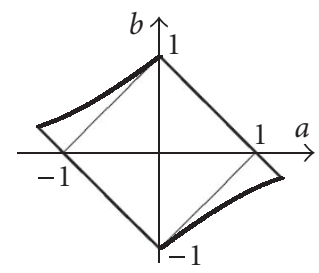

(a)

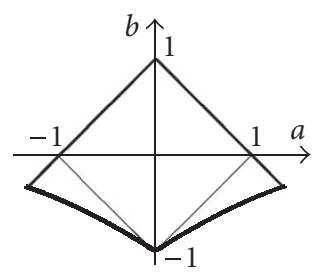

(b)

Figure 1.1. The stability domains of (1.2). (a) $k$ is odd, $k>1$. (b) $k$ is even. The Cohn domain boundary $|a|+|b|=1$ are shown both in (a), (b).

following lines:

(I) $a+b=1$,

(II) $a=-\sin k \omega / \sin (k-1) \omega, b=(-1)^{k+1} \sin \omega / \sin (k-1) \omega$,

(III) $(-1)^{k} b-a=1$,

(IV) $a=\sin k \omega / \sin (k-1) \omega, b=-\sin \omega / \sin (k-1) \omega$,

where $\omega$ varies between 0 and $\pi / k$.

It follows from Cohn's results [3] that (1.2) is asymptotically stable provided that

$$
|a|+|b|<1
$$

A special case of (1.2), namely, the scalar equation $x_{n}=x_{n-1}+b x_{n-k}$, was studied by Levin and May [9]. They established that the equation is asymptotically stable if and only if $2 \sin (\pi / 2(2 k-1))>-b>0$. Scalar equation with two delays $x_{n}=a x_{n-m}+b x_{n-k}$, more compound than (1.2), was investigated by Dannan [5], Kipnis and Nigmatulin [7], and Nikolaev $[13,14]$.

The matrix equation

$$
x_{n}=x_{n-1}+B x_{n-k},
$$

where $B$ is an $(m \times m)$ real matrix, $x_{n}: \mathbb{N} \rightarrow \mathbb{R}^{m}$, a positive integer $k$ is a delay, was investigated by Levitskaya [10]. She transferred Rekhlitskii's result [16] about the differential equation $\dot{x}=A x(t-\tau)$, where a positive $\tau$ is a delay, $A$ is a real matrix, to the difference equations. She established that (1.4) is asymptotically stable if and only if any eigenvalue of the matrix $B$ lies inside the oval of the complex plane bounded by a curve

$$
\Gamma=\left\{z \in \mathbb{C}: z=2 i \sin \frac{\varphi}{2 k-1} e^{i \varphi},-\frac{\pi}{2} \leq \varphi \leq \frac{\pi}{2}\right\}
$$

Our first problem is to obtain a characteristic equation for (1.1). Second problem is to obtain the necessary and sufficient condition in terms of the eigenvalues location of the matrix $B$ for the asymptotic stability of the equation

$$
x_{n}=-x_{n-1}+B x_{n-k},
$$


where $B$ is a real $(m \times m)$ matrix. Further, we give a sufficient condition for the asymptotic stability of (1.1) similar with the Cohn condition (1.3). Finally, we give explicit sufficient stability conditions for (1.1) in cases $A \simeq I$ and $A \simeq-I$, that is, when (1.1) looks like (1.4) and (1.6), respectively.

\section{Characteristic equation of (1.1)}

The following theorem deals with the stability of the linear system:

$$
x_{n}=\sum_{i=1}^{k} A_{i} x_{n-i}
$$

where $A_{i}$ are $(m \times m)$ real matrixes $(i=1,2, \ldots, k), x_{n}: \mathbb{N} \rightarrow \mathbb{R}^{m}$. The equations of type (2.1) in a Banach space were investigated in [1].

Theorem 2.1. Equation (2.1) is asymptotically stable if and only if all roots of the equation

$$
\operatorname{det}\left(I z^{k}-\sum_{i=1}^{k} A_{i} z^{k-i}\right)=0
$$

lie inside the unit disk. If at least one root of (2.2) lies outside of the unit disk, then (2.1) is unstable.

Proof. Consider a generating function $x(z)=\sum_{n=0}^{\infty} x_{n} z^{n}$ of the sequence $x_{n}(n \geq 0)$. For the solution of (2.1), the following equality holds:

$$
x(z)-\sum_{i=1}^{k} A_{i}\left(\sum_{j=0}^{i-1} x_{j-i} z^{j}+x(z) z^{i}\right)=0 .
$$

Here $x_{-1}, \ldots, x_{-k}$ are the initial conditions. From (2.3), we have $\left(I-\sum_{i=1}^{k} A_{i} z^{i}\right) x(z)=$ $F(z)$, where $F(z)$ is an $(m \times 1)$ matrix whose elements are polynomials. Hence,

$$
x(z)=\frac{1}{\operatorname{det}\left(I-\sum_{i=1}^{k} A_{i} z^{i}\right)} Q(z),
$$

where $Q(z)$ is another $(m \times 1)$ matrix whose elements are polynomials. If all roots of $(2.2)$ lie inside the unit disk, then all roots of the denominator of (2.4) lie outside the unit disk, and consequently, $x(z)$ may be expanded in a power series with the radius of convergence greater than 1 . Hence, $x_{n}$ tends to 0 exponentially with $n \rightarrow \infty$ regardless of the initial conditions. It follows that $(2.1)$ is asymptotically stable.

Let there exist a root $z$ of (2.2), such that $|z| \geq 1$. We will seek for the solution of (2.1) in the form $x_{n}=D z^{n}$, where $D \in \mathbb{C}^{m}, D \neq 0$. Then we obtain $\left(I z^{k}-\sum_{i=1}^{k} A_{i} z^{k-i}\right) D=0$. This system is degenerate. Hence, there exists nonzero solution $D$, and $x_{n}=D z^{n}$ does not tend to zero as $n \rightarrow \infty$. Both real sequences $x_{n}=\operatorname{Re} D z^{n}$ and $x_{n}=\operatorname{Im} D z^{n}$ are solutions of (2.1), and at least one of them does not tend to zero. Hence, (2.1) is not asymptotically 
stable. If, in addition, $|z|>1$, then the solution $x_{n}=D z^{n}$ is unbounded. At least one of the real sequences $x_{n}=\operatorname{Re} D z^{n}$ or $x_{n}=\operatorname{Im} D z^{n}$ is unbounded, and (2.1) is unstable.

Corollary 2.2. Equation (1.1) is asymptotically stable if and only if all roots of the equation

$$
\operatorname{det}\left(B+A z^{k-1}-I z^{k}\right)=0
$$

lie inside the unit disk. If at least one root of (2.5) lies outside the unit disk, then (1.1) is unstable.

A question arises: can we formulate a necessary and sufficient condition for the asymptotic stability of (1.1) in terms of restrictions on the eigenvalues of matrixes $A$ and $B$ ? The answer is no, as indicated by the following example.

Example 2.3. Consider the equation $x_{n}=A x_{n-1}+B x_{n-3}$, where $B=\left(\begin{array}{cc}-0.1 & 0.3 \\ 0.1 & 0.2\end{array}\right)$. In case $A=$ $\left(\begin{array}{cc}0.1 & -0.6 \\ -0.7 & 0.2\end{array}\right)$, the equation is asymptotically stable by the Corollary 2.2. However, if $A=$ $\left(\begin{array}{cc}-0.2 & 0.6 \\ 0.5 & 0.5\end{array}\right)$, then it is unstable, although eigenvalues of the matrix $A$ are $\lambda_{1}=0.8, \lambda_{2}=-0.5$ in both cases.

If $k>1$ and $|b|>1$, then the scalar equation (1.2) is unstable (Figure 1.1). We obtain a similar result for the matrix equations (1.1) and (2.1).

Theorem 2.4. If $\left|\operatorname{det} A_{k}\right|>1$, then (2.1) is unstable.

Proof. The characteristic equation (2.2) has the form

$$
\operatorname{det}\left(I z^{k}-\sum_{i=1}^{k} A_{i} z^{k-i}\right) \equiv P(z) \equiv \sum_{i=0}^{k m} a_{i} z^{i}=0,
$$

where $a_{k m}=1$. We put $z=0$ in (2.6) and obtain $\left|a_{0}\right|=\left|\operatorname{det} A_{k}\right|>1$. Hence, there exists a root of the polynomial $P(z)$ outside of the unit disk, since $\left|a_{0}\right|$ is the modulus of product of all roots of $P(z)$.

Corollary 2.5. If $k>1$ and $|\operatorname{det} B|>1$, then (1.1) is unstable.

\section{Stability ovals for (1.6)}

THeOREM 3.1. The system (1.6) is asymptotically stable if and only if all eigenvalues of the matrix $B$ lie inside the region of the complex plane bounded by the curve

$$
\Gamma=\left\{z \in \mathbb{C}: z=(-1)^{k} 2 i \sin \frac{\varphi}{2 k-1} e^{i \varphi},-\frac{\pi}{2} \leq \varphi \leq \frac{\pi}{2}\right\}
$$

Proof. We first consider the scalar equation

$$
x_{n}=-x_{n-1}+\lambda x_{n-k}
$$

where $\lambda \in \mathbb{C}$. The characteristic equation for (3.2) is

$$
z^{k}+z^{k-1}=\lambda
$$


Writing $z=-v$ in (3.3) gives

$$
v^{k}-v^{k-1}=(-1)^{k} \lambda
$$

But all roots of the equation

$$
z^{k}-z^{k-1}=\lambda
$$

lie inside the unit disk if and only if $\lambda$ lies inside the region of the complex plane bounded by the curve (1.5). A proof of this fact had been given in [10, Theorem 1]. Therefore, all roots of (3.4), as well as (3.3), lie inside the unit disk if and only if $\lambda$ lies inside the oval (3.1).

Return to the matrix equation (1.6). It follows from Corollary 2.2 that the characteristic equation for the system (1.6) is

$$
\operatorname{det}\left(B-I\left(z^{k}+z^{k-1}\right)\right)=0 .
$$

Let $\lambda_{1}, \lambda_{2}, \ldots, \lambda_{m}$ be the eigenvalues of $B$. Consider the equation (cf. (3.3))

$$
z^{k}+z^{k-1}=\lambda_{i}, \quad i=1,2, \ldots, m
$$

If each root of any equation (3.7) lies inside the unit disk, then each solution of (3.6) lies inside the unit disk, and conversely. The inclusion of all solutions of (3.7) in the unit disk is the condition for number $\lambda_{i}$ to belong to the interior of oval (3.1). Thus, system (1.6) is asymptotically stable if and only if all eigenvalues of $B$ lie inside the oval bounded by the curve (3.1).

Stability ovals for (1.6) are displayed in Figure 3.1.

Projection of the results of Theorem 3.1 onto the real axis gives the following addition to the classical result of Levin and May [9], mentioned in the introduction.

Corollary 3.2. The scalar equation (3.2) with $\lambda \in \mathbb{R}$ is asymptotically stable if and only if

$$
2 \sin \frac{\pi}{2(2 k-1)}>(-1)^{k+1} \lambda>0
$$

Remark 3.3. If $k=1$, then the stability oval is the disk of radius 1 centered at $(1+0 i)$. If $k$ is odd in (1.6), then stability oval is located in the right half-plane, if $k$ is even, then it is located in the left half-plane. Therefore, it is necessary for asymptotic stability of (1.6) that the condition $\operatorname{Re} \lambda_{i}>0$ with $k$ odd and the condition $\operatorname{Re} \lambda_{i}<0$ with $k$ even hold. Here $\lambda_{i}(1 \leq i \leq m)$ are the eigenvalues of $B$. Compare it with scalar equation (1.2) and its stability domain in Figure 1.1. We see that with $a=-1$ in (1.2), the condition $b>0$ is necessary for asymptotic stability with $k$ odd, while $b<0$ is necessary with $k$ even. The similarity is evident.

Corollary 3.4. If there exist eigenvalues $\lambda_{1}, \lambda_{2}$ of $B$, such that $\operatorname{Re} \lambda_{1}>0, \operatorname{Re} \lambda_{2}<0$, then (1.6) is unstable with any $k$. 


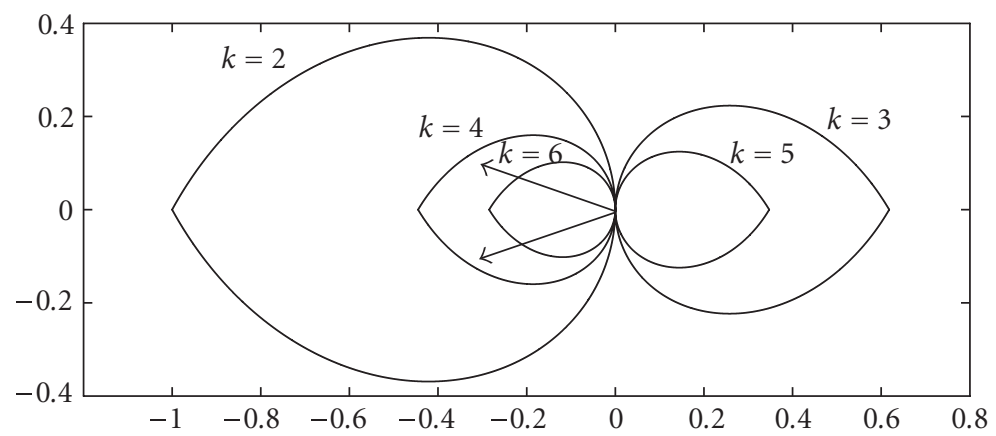

Figure 3.1. Stability ovals for the system (1.6). The arrows represent the eigenvalues of a matrix $B$ in Example 3.6.

Now we are in a position to strengthen Corollary 2.5 for (1.4) and (1.6).

THeOREm 3.5. If the system (1.4) (the system (1.6)) is asymptotically stable, then all eigenvalues of $B$ lie inside the unit disk.

Proof. It is sufficient to consider the stability ovals (1.5) and (3.1) and to remark that $|2 \sin (\pi / 2(2 k-1))| \leq 1$ for $k>1$.

Example 3.6. Consider (1.6) with $B=\left(\begin{array}{cc}0.5 & 1.3 \\ -0.5 & -1.1\end{array}\right)$. Eigenvalues of the matrix $B$ are $\lambda_{1,2}=$ $-0.3 \pm 0.1 i$. Configuration of the $\lambda_{1}, \lambda_{2}$, and the stability ovals (see Figure 3.1) indicates that the equation is asymptotically stable for $k=2,4$ and unstable for $k=1,3$, and for any $k>4$.

\section{Explicit stability conditions for (1.1)}

In what follows, $\|\cdot\|$ is any matrix norm which satisfies the following conditions:

(I) $\|A\| \geq 0$, and $\|A\|=0$ if and only if $A=0$,

(II) for each $c \in \mathbb{R},\|c A\|=|c| \cdot\|A\|$,

(III) $\|A+B\| \leq\|A\|+\|B\|$,

(IV) $\|A B\| \leq\|A\| \cdot\|B\|$ for all $(m \times m)$ matrices $A, B$.

In addition, matrix norm should be concordant with the vector norm $\|\cdot\|_{*}$, that is,

$$
\|A x\|_{*} \leq\|A\| \cdot\|x\|_{*}
$$

for all $x \in \mathbb{R}^{m}$ and any $(m \times m)$ matrix $A$.

For real $(m \times m)$ matrix $A$, we define, as usual, $\|A\|_{1}=\max _{1 \leq j \leq m} \sum_{i=1}^{m}\left|a_{i j}\right|$ and $\|A\|_{\infty}=$ $\max _{1 \leq i \leq m} \sum_{j=1}^{m}\left|a_{i j}\right|$.

We will give a Cohn-type sufficient stability condition [3] for (2.1) (see also [2, Theorem 2.1] and [11, Theorem 2]).

Theorem 4.1. If $\sum_{i=1}^{k}\left\|A_{i}\right\|<1$, then (2.1) is asymptotically stable. 
Proof. Assume that $\max \left(\left\|x_{-1}\right\|, \ldots,\left\|x_{-k}\right\|\right)=M$ and $\sum_{i=1}^{k}\left\|A_{i}\right\|=b<1$. Sequentially for $n=0,1, \ldots, k-1$, we have

$$
\left\|x_{n}\right\|=\left\|\sum_{i=1}^{k} A_{i} x_{n-i}\right\| \leq \sum_{i=1}^{k}\left\|A_{i}\right\| M=b M<M .
$$

Then for $n=k, k+1, \ldots, 2 k-1$, we derive sequentially

$$
\left\|x_{n}\right\|=\left\|\sum_{i=1}^{k} A_{i} x_{n-i}\right\| \leq \sum_{i=1}^{k}\left\|A_{i}\right\| b M=b^{2} M<b M .
$$

Furthermore, for $n \geq r k, r \in \mathbb{N}$, we deduce similarly that $\left\|x_{n}\right\| \leq b^{r+1} M$.

Next Corollary gives a delay-independent Cohn-type stability condition for (1.1).

Corollary 4.2. If

$$
\|A\|+\|B\|<1
$$

then (1.1) is asymptotically stable.

Example 4.3. Consider (1.1) with $A=\left(\begin{array}{cc}-0.5 & 0 \\ 0.4 & 0.1\end{array}\right), B=\left(\begin{array}{cc}0.3 & -0.1 \\ -0.2 & 0.2\end{array}\right)$. We have $\|A\|_{1}+\|B\|_{1}=$ $1.4>1$, but $\|A\|_{\infty}+\|B\|_{\infty}=0.9<1$. By Corollary 4.2 , the equation is asymptotically stable for any delay $k$.

Some additional domains to the Cohn domain for scalar variant of (2.1) were described in [2, Theorem 2.3, Corollary 2.4] employing the Halanay-type inequalities. In the spirit of [2] and earlier work [4], we will give a sufficient stability condition of (1.1), additional to Cohn-type condition (4.4). The following result is convenient for application to (1.1) when $\|A-I\| \ll 1$ and $\|B\| \ll 1$.

THeorem 4.4. If

$$
\|A+B\|+(k-1)\|B\|(\|A-I\|+\|B\|)<1,
$$

then (1.1) is asymptotically stable.

Proof. Let us rewrite (1.1),

$$
\begin{aligned}
x_{n} & =(A+B) x_{n-1}-B\left(x_{n-1}-x_{n-k}\right) \\
& =(A+B) x_{n-1}-B \sum_{i=1}^{k-1}\left(x_{n-i}-x_{n-i-1}\right) \\
& =(A+B) x_{n-1}-B \sum_{i=1}^{k-1}\left((A-I) x_{n-i-1}+B x_{n-k-i}\right) .
\end{aligned}
$$

Combining Theorem 4.1 with (4.6), we obtain the conclusion of the theorem. 
Example 4.5. Consider (1.1) with $A=\left(\begin{array}{cc}1.01 & -0.02 \\ 0 & 1.01\end{array}\right)$ and $B=\left(\begin{array}{cc}-0.2 & 0 \\ 0.01 & -0.2\end{array}\right)$. It is easily seen that $\|A\|+\|B\|>1$ for any norm $\|\cdot\|$. The Cohn-type condition (4.4) cannot be applied. However, $\|A+B\|_{1}+(k-1)\|B\|_{1}\left(\|A-I\|_{1}+\|B\|_{1}\right)=0.83+(k-1) 0.0504$. Hence, Theorem 4.4 guarantees asymptotic stability of $(1.1)$ for $k=1,2,3,4$. Additional calculations, based on Corollary 2.2, give instability for $k>4$.

A lot of explicit stability conditions for scalar equations (see, e.g., $[2,11,4,12])$ can be translated to the language of linear systems (1.1). However, we will indicate a new phenomenon. We introduce an additional stability condition for (1.1) depending on whether the delay $k$ is odd or even. The condition is convenient for applications to (1.1) when $\|A+I\| \ll 1$ and $\|B\| \ll 1$.

THEOREM 4.6. If

$$
\left\|A+(-1)^{k+1} B\right\|+(k-1)\|B\|(\|A+I\|+\|B\|)<1,
$$

then (1.1) is asymptotically stable.

Proof. Assume $k$ is odd. Let us rewrite (1.1) as

$$
\begin{aligned}
x_{n} & =(A+B) x_{n-1}-B\left(x_{n-1}-x_{n-k}\right) \\
& =(A+B) x_{n-1}-B \sum_{i=1}^{k-1}(-1)^{i+1}\left((A+I) x_{n-i-1}+B x_{n-k-i}\right) .
\end{aligned}
$$

The conclusion of the theorem is a consequence of Theorem 4.1 and (4.8).

Now suppose that $k$ is even. We rewrite (1.1) as

$$
\begin{aligned}
x_{n} & =(A-B) x_{n-1}+B\left(x_{n-1}+x_{n-k}\right) \\
& =(A-B) x_{n-1}+B \sum_{i=1}^{k-1}(-1)^{i+1}\left((A+I) x_{n-i-1}+B x_{n-k-i}\right) .
\end{aligned}
$$

The proof is completed by the same arguments as in the preceding case.

Example 4.7. Consider (1.1) with $A=\left(\begin{array}{cc}-1.01 & -0.02 \\ 0 & -1.01\end{array}\right)$ and $B=\left(\begin{array}{cc}-0.2 & 0 \\ 0.01 & -0.2\end{array}\right)$ (cf. Example 4.5). The Cohn-type condition (4.4) cannot be applied. For odd values of $k$, Theorem 4.6 is useless: $\|A+B\|>1$ for any norm $\|\cdot\|$, hence (4.7) fails when $k$ is odd. For even values of $k$, we have $\|A-B\|_{1}+(k-1)\|B\|_{1}\left(\|A+I\|_{1}+\|B\|_{1}\right)=0.83+(k-1) 0.0504$. Theorem 4.6 now implies asymptotic stability of the equation for $k=2$ and $k=4$. Additional calculations of the roots of characteristic equation (2.5) give instability for $k=1, k=3$, and for any $k>4$.

\section{Acknowledgments}

This work was partially supported by the Russian Foundation for Basic Research, Grants 04-01-96069 and 07-01-96065. The authors thank V. Karachik, A. Makarov, S. Pinchuk, L. Pesin, and D. Scheglov for their helpful discussions. 


\section{References}

[1] L. Berezansky and E. Braverman, On exponential dichotomy, Bohl-Perron type theorems and stability of difference equations, Journal of Mathematical Analysis and Applications 304 (2005), no. 2, 511-530.

[2] L. Berezansky, E. Braverman, and E. Liz, Sufficient conditions for the global stability of nonautonomous higher order difference equations, Journal of Difference Equations and Applications 11 (2005), no. 9, 785-798.

[3] A. Cohn, Über die Anzahl der Wurzeln einer algebraischen Gleichung in einem Kreise, Mathematische Zeitschrift 14 (1922), no. 1, 110-148.

[4] K. L. Cooke and I. Györi, Numerical approximation of the solutions of delay differential equations on an infinite interval using piecewise constant arguments, Computers \& Mathematics with Applications 28 (1994), no. 1-3, 81-92.

[5] F. M. Dannan, The asymptotic stability of $x(n+k)+a x(n)+b x(n-l)=0$, Journal of Difference Equations and Applications 10 (2004), no. 6, 589-599.

[6] J. Diblík and D. Ya. Khusainov, Representation of solutions of discrete delayed system $x(k+1)=$ $A x(k)+B x(k-m)+f(k)$ with commutative matrices, Journal of Mathematical Analysis and Applications 318 (2006), no. 1, 63-76.

[7] M. Kipnis and R. M. Nigmatulin, Stability of trinomial linear difference equations with two delays, Automation and Remote Control 65 (2004), no. 11, 1710-1723.

[8] S. A. Kuruklis, The asymptotic stability of $x_{n+1}-a x_{n}+b x_{n-k}=0$, Journal of Mathematical Analysis and Applications 188 (1994), no. 3, 719-731.

[9] S. A. Levin and R. M. May, A note on difference-delay equations, Theoretical Population Biology 9 (1976), no. 2, 178-187.

[10] I. S. Levitskaya, A note on the stability oval for $x_{n+1}=x_{n}+A x_{n-k}$, Journal of Difference Equations and Applications 11 (2005), no. 8, 701-705.

[11] E. Liz and J. B. Ferreiro, A note on the global stability of generalized difference equations, Applied Mathematics Letters 15 (2002), no. 6, 655-659.

[12] E. Liz and M. Pituk, Asymptotic estimates and exponential stability for higher-order monotone difference equations, Advances in Difference Equations 2005 (2005), no. 1, 41-55.

[13] Yu. P. Nikolaev, The set of stable polynomials of linear discrete systems: its geometry, Automation and Remote Control 63 (2002), no. 7, 1080-1088.

[14] The geometry of D-decomposition of a two-dimensional plane of arbitrary coefficients of the characteristic polynomial of a discrete system, Automation and Remote Control 65 (2004), no. 12, 1904-1914.

[15] V. G. Papanicolaou, On the asymptotic stability of a class of linear difference equations, Mathematics Magazine 69 (1996), no. 1, 34-43.

[16] Z. I. Rekhlitskii, On the stability of solutions of certain linear differential equations with a lagging argument in the Banach space, Doklady Akademii Nauk SSSR 111 (1956), 770-773 (Russian).

Mikhail Kipnis: Department of Mathematics, Chelyabinsk State Pedagogical University, 69 Lenin Avenue, Chelyabinsk 454080, Russia

E-mail address: mkipnis@cspu.ru

Darya Komissarova: Department of Mathematics, Southern Ural State University, 76 Lenin Avenue, Chelyabinsk 454080, Russia

E-mail address: dasha@math.susu.ac.ru 